\title{
E-cadherin Is Important for the Maintenance of Intestinal Epithelial Homeostasis Under Basal and Inflammatory Conditions
}

\author{
Michael Schnoor ${ }^{1}$
}

Published online: 14 March 2015

(C) Springer Science+Business Media New York 2015

The intestinal epithelial layer forms an important barrier that protects underlying tissues from bacteria within the gut lumen. This single monolayer is comprised of epithelial cells that are connected by intercellular junctions. The most apical junctions are tight junctions (TJ) followed by adherens junctions (AJ) and desmosomes. AJ mediate cellcell adhesion mainly via E-cadherin. Cell junctions are dynamic structures that continuously need to respond to stimuli that may stabilize or destabilize inter-epithelial cell contacts. Inflammatory cytokines activate mediators of signaling pathways such as small GTPases that destabilize junctions by internalization or proteolysis of junction molecules, including E-cadherin [1-3]. Such processing disconnects junctions from the actin cytoskeleton, consequently destabilizing cell contacts that facilitate invasion of pathogens to further trigger inflammation. If not controlled or resolved properly, such destabilization may lead to the development of chronic inflammatory conditions such as inflammatory bowel diseases (IBD), including ulcerative colitis and Crohn's disease. IBD are characterized by increased intestinal epithelial permeability, painful cramping, bloody diarrhea, and weight loss [4]. Since the pathogenesis of IBD and curative treatments remain elusive, the study of the pathologic derangements of molecular mechanisms associated with IBD is important in the development of novel treatment strategies. In this respect transmembranal junctional adhesion molecules are

Michael Schnoor

mschnoor@cinvestav.mx

1 Department of Molecular Biomedicine, Center for Research and Advanced Studies of the National Polytechnic Institute (CINVESTAV-IPN), Av. IPN 2508, San Pedro Zacatenco, GAM, 07360 Mexico City, Mexico important because they directly mediate cell-cell adhesion and thus stabilize the entire epithelial monolayer. For example, the physiological significance of E-cadherin has been investigated intensively using in vivo and in vitro models simulating the clinical and pathological manifestations of IBD [5]. The use of E-cadherin knockout (KO) and knockin mouse models in the study of tissue-specific effects of E-cadherin loss or replacement has recently been reviewed [6]. Of note, total E-cadherin $\mathrm{KO}$ in mice is embryonically lethal [7]. Intestinal-specific deletion of the floxed CDH1 gene using Villin-Cre mice was associated with death within $24 \mathrm{~h}$ after birth [8]. Targeted E-cadherin deletion was induced in adult mice using a tamoxifen-induced deletion that was associated with bloody diarrhea with death attributed to epithelial shedding [9]. These data highlight the importance of E-cadherin for intestinal epithelial homeostasis even under basal conditions. In vitro, inflammatory conditions, simulated by IFN- $\gamma$ treatment of T84 cells, reduced surface expression of E-cadherin, enhanced AJ disassembly, and overall destabilized the epithelial monolayer [10]. Yet, it remained elusive how the induced loss of E-cadherin would affect the development of colitis in vivo (Fig. 1).

In this issue of Digestive Diseases and Sciences, Grill et al. report that acute loss of E-cadherin aggravates experimental colitis [11]. Using a mouse model of combined tamoxifen injections to induce loss of E-cadherin and $3.5 \%$ dextran sulfate sodium (DSS) in drinking water to induce colitis, the authors demonstrate that mice with DSS colitis and acute loss of E-cadherin had more frequent bloody stools and an increased hematocrit. Histological analysis of colon tissues revealed signs of severe inflammation and tissue damage, including increased neutrophil infiltration in E-cadherin $\mathrm{KO}$ mice compared to controls after DSS treatment. Junctional localization of the TJ 


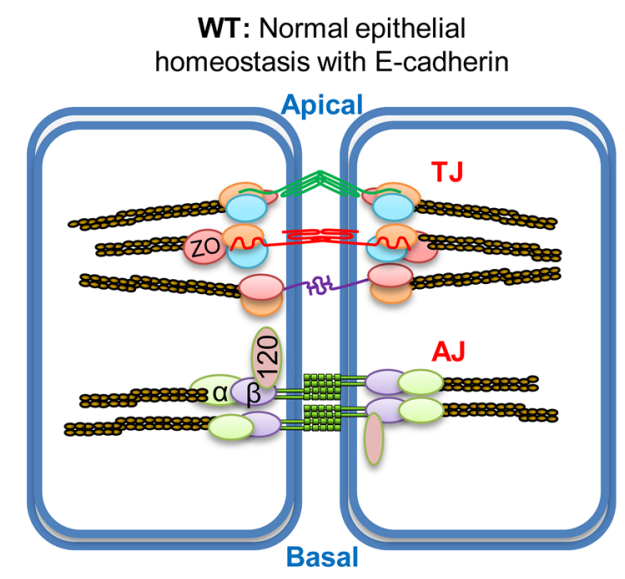

WT + DSS: E-cad internalization, AJ disassembly and diarrhea

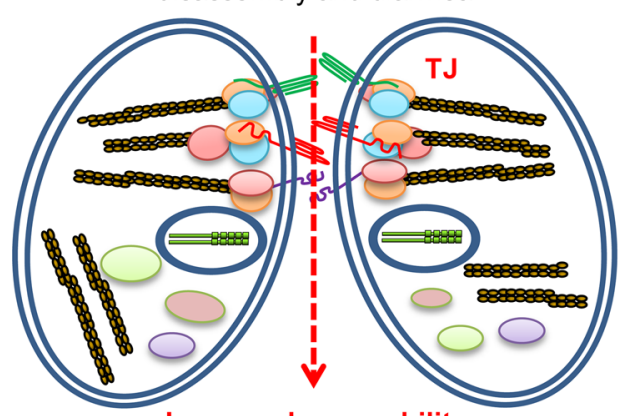

Increased permeability

Fig. 1 Effects of E-cadherin deficiency in the adult intestine under basal and inflammatory conditions. Under basal conditions, intestinal WT epithelium is characterized by a stable barrier formed by tight junctions (TJ), adherens junctions (AJ), and more basally located desmosomes (not depicted for simplicity), which are connected to the actin cytoskeleton by adaptor molecules such as members of the zonula occludens $(\mathrm{ZO})$ or catenin $(\alpha, \beta, \mathrm{p} 120)$ families. Induced E-cadherin deficiency (E-cad KO) is associated with the loss of AJ,

protein occludin was reduced after DSS treatment, as expected, but by similar amounts in control and E-cadherin KO mice, suggesting that the loss of E-cadherin per se does not necessarily affect $\mathrm{TJ}$ architecture. This is of particular importance because mice deleted for TJ proteins such as occludin or junctional adhesion molecule (JAM)-A do not show signs of spontaneous colitis, suggesting that E-cadherin is more necessary for epithelial barrier integrity in vivo than single $\mathrm{TJ}$ components. Interestingly, E-cadherin KO mice treated with DSS had more pronounced evidence of epithelial regeneration, thus confirming earlier findings of increased proliferation in the absence of E-cadherin [5]. This observation is logical, given that the loss of E-cadherin reduces adhesion and increases cellular migratory properties, for example during epithelial-mesenchymal transition, which could relate to a higher risk of developing epithelial cancer in the colon of E-cadherindeficient mice [5].
E-cad KO: AJ disruption

leading to bloody diarrhea and death

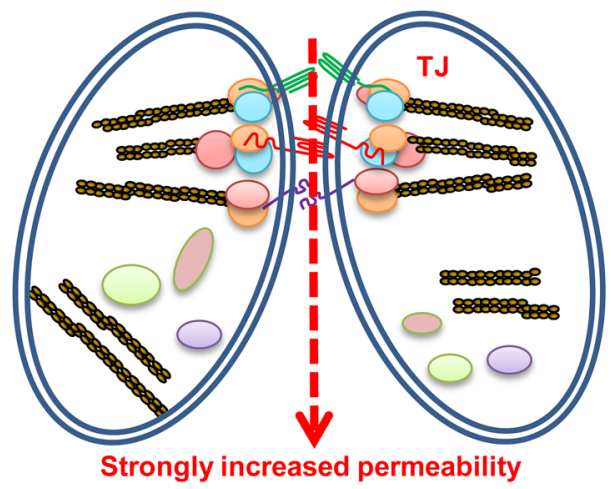

E-cad KO + DSS: Exaggerated inflammation and epithelial damage cause more frequent death

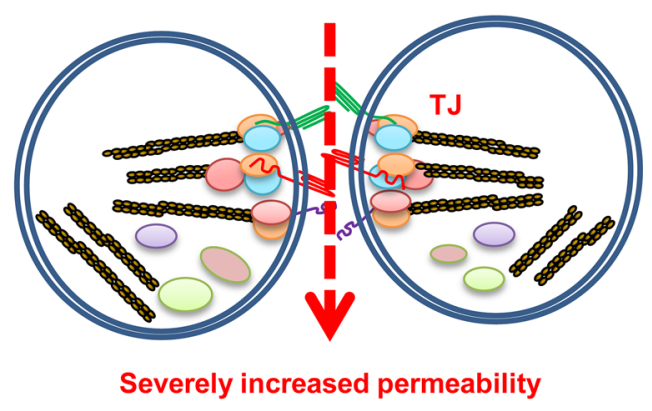

altered cell morphology, and strongly increased epithelial barrier permeability as manifested by bloody diarrhea and early death. WT mice treated with DSS have similar characteristics as do E-cad KO, such as junction disassembly via internalization of adhesion molecules and increased barrier permeability. E-cad KO mice treated with DSS show even more pronounced signs of inflammation and tissue damage leading to severe bloody diarrhea, weight loss, and earlier death compared to WT mice treated with DSS

E-cadherin was also important for clearance of pathogens, suggesting its importance in the intestinal immune system. Defective pathogen clearance and bacterial translocation in the absence of E-cadherin may thus be a key factor triggering the progression of IBD.

In summary, the data shown by Grill and colleagues highlight the prominent contribution of E-cadherin toward epithelial barrier maintenance during inflammation. Even though these results could be predicted based on data documenting the importance of E-cadherin for barrier development and homeostasis under basal conditions, direct evidence to support this notion was missing until now. This new evidence is important since intestine-specific $\mathrm{KO}$ mice did have diarrhea and died early but lacked other characteristics of acute experimental colitis induced by DSS. Although it is known that E-cadherin is downregulated in tissue biopsies of IBD patients, it will be necessary to verify whether the loss of E-cadherin precedes the onset of 
IBD or whether this is rather a response to the chronic inflammatory milieu in the IBD intestinal mucosa that contributes to disease progression. Another mechanism that could relate to the severe epithelial phenotype is the loss of the cadherin/catenin complex and links to the cytoskeleton. For example, p120-catenin KO mice are characterized by disturbed epithelial adhesion, increased inflammation, mucosal erosions, and bloody stools, effects similar to what has been observed in the study by Grill and colleagues [12]. Thus, it will be important to investigate in future studies how colitis and loss of E-cadherin affect catenin functions and the cytoskeleton, which are major factors for controlling epithelial barrier integrity.

\section{References}

1. Citalan-Madrid AF, Garcia-Ponce A, Vargas-Robles H, et al. Small GTPases of the Ras superfamily regulate intestinal epithelial homeostasis and barrier function via common and unique mechanisms. Tissue Barriers. 2013;1:e26938.

2. Ivanov AI, Naydenov NG. Dynamics and regulation of epithelial adherens junctions: recent discoveries and controversies. Int Rev Cell Mol Biol. 2013;303:27-99.

3. Nava P, Kamekura R, Nusrat A. Cleavage of transmembrane junction proteins and their role in regulating epithelial homeostasis. Tissue Barriers. 2013;1:e24783.
4. Xavier RJ, Podolsky DK. Unravelling the pathogenesis of inflammatory bowel disease. Nature. 2007;448:427-434.

5. Mehta S, Nijhuis A, Kumagai T, et al. Defects in the adherens junction complex (E-cadherin/beta-catenin) in inflammatory bowel disease. Cell Tissue Res. 2014. doi:10.1007/s00441-0141994-6.

6. Schneider MR, Kolligs FT. E-cadherin's role in development, tissue homeostasis and disease: insights from mouse models: tissue-specific inactivation of the adhesion protein E-cadherin in mice reveals its functions in health and disease. Bioessays. 2015;37:294-304.

7. Larue L, Ohsugi M, Hirchenhain J, et al. E-cadherin null mutant embryos fail to form a trophectoderm epithelium. Proc Natl Acad Sci USA. 1994;91:8263-8267.

8. Bondow BJ, Faber ML, Wojta KJ, et al. E-cadherin is required for intestinal morphogenesis in the mouse. Dev Biol. 2012;371:1-12.

9. Schneider MR, Dahlhoff M, Horst D, et al. A key role for E-cadherin in intestinal homeostasis and Paneth cell maturation. PLoS One. 2010;5:e14325.

10. Smyth D, Leung G, Fernando M, et al. Reduced surface expression of epithelial E-cadherin evoked by interferon-gamma is Fyn kinase-dependent. PLoS One. 2012;7:e38441.

11. Grill JI, Neumann J, Hiltwein F, et al. Intestinal E-cadherin deficiency aggravates dextran sodium sulfate-induced colitis. Dig Dis Sci. (Epub ahead of print). doi:10.1007/s10620-015-3551-x.

12. Smalley-Freed WG, Efimov A, Burnett PE, et al. p120-catenin is essential for maintenance of barrier function and intestinal homeostasis in mice. J Clin Invest. 2010;120:1824-1835. 